\title{
Facet-Dependent Changes in Crystallinity and Atom Exchange of Hematite Nanocrystals during Fe(II)- Accelerated Recrystallization
}

\author{
FEI WU ${ }^{1,2}$, CHENGSHUAI LIU $^{1,2^{*}}$ \\ ${ }^{1}$ State Key Laboratory of Environmental Geochemistry, Institute \\ of Geochemistry, Chinese Academy of Sciences, Guiyang \\ 550081, PR China \\ ${ }^{2}$ Guangdong Key Laboratory of Integrated Agroenvironmental \\ Pollution Control and Management, Guangdong Institute of \\ Eco-environmental Science \& Technology, Guangzhou \\ 510650, PR China \\ (*correspondence: liuchengshuai@vip.gyig.ac.cn)
}

It is well established that the chemical and catalytic activity of crystalline materials can strongly depend on the types and proportions of their exposed facets. Hematite $\left(\alpha-\mathrm{Fe}_{2} \mathrm{O}_{3}\right)$ is one such material which exhibits various crystal morphologies include platy, rhombohedral, and rounded, often based upon (001), (012), (110), and (104) facets. To obtain a detailed understanding of facetspecific mechanisms and atom exchange in the $\mathrm{Fe}(\mathrm{II})$-accelerated recrystallization of hematite, we used two different structurally well-defined hematite nanoparticle morphologies reacted with ${ }^{57} \mathrm{Fe}(\mathrm{II}) a q$ as a tracer at neutral $\mathrm{pH}$. After reaction with ${ }^{57} \mathrm{Fe}(\mathrm{II})$, we observed partial suppression of the Morin transition of hematite to below $13 \mathrm{~K}$. This is significantly lower than the Morin temperature (TM) of $\sim 210 \mathrm{~K}$ measured for hematite nanoparticles. And the abundance of weakly ferromagnetic (WF) phase at $13 \mathrm{~K}$ for nanoplates dominated by $\{001\}$ facets and nancubes dominated by $\{012\}$ facets were $73.6 \%$ and $38.0 \%$ (based on the Mössbauer spectra area), respectively. $\{001\}$ facets induced more iron atom exchange than $\{012\}$ facets, indicating that the $\mathrm{Fe}(\mathrm{II})-$ accelerated recrystallization process was mediated by exposed facets of hematite. And facet-spefic differences appear to be not directly linked with the simple aerial cation site density, while with their extent of under-coordination. High resolution transmission electron microscopy (HR-TEM) and fast Fourier transforms (FFT) showed that the $\{001\}$ facets has the same lattice spacing $(2.5 \AA)$ before and after reacted with $\mathrm{Fe}(\mathrm{II})$, while the lattice spacing of $\{012\}$ facets changed from $4.6 \AA$ to $3.7 \AA$. Our results resolving $\mathrm{Fe}(\mathrm{II})-\mathrm{Fe}(\mathrm{III})$ reaction fronts across multi-faceted crystals provide a clear correlation between recrystallization and particle surface structure. 